\section{G377 THE ROLE OF CARDIOPULMONARY EXERCISE TESTING IN CHILDREN WITH CYSTIC FIBROSIS}

${ }^{1} \mathrm{E}$ Weir, ${ }^{1} \mathrm{P}$ Burns, ${ }^{2} \mathrm{D}$ Young, ${ }^{1} \mathrm{JY}$ Paton, ${ }^{1} \mathrm{~A}$ Devenny. ${ }^{1}$ Respiratory Paediatrics, Royal Hospital for Sick Children, Glasgow, UK; ${ }^{2}$ Department of Mathematics and Statistics, University of Strathclyde, Glasgow, UK

\subsection{6/archdischild-2015-308599.332}

Introduction Cardiopulmonary exercise testing (CPET) is the gold standard test of aerobic fitness in Cystic Fibrosis (CF). CPET is measured by peak oxygen uptake (VO2 peak) during maximal exercise. It can be used to identify the cause of exercise intolerance, prescribe exercise programmes, evaluate therapeutic interventions ${ }^{1}$ and predict prognosis. ${ }^{2}$ The American Thoracic Society defines a normal VO2 peak as $>84 \%$ predicted. ${ }^{3}$ In our centre, CPET was included in the CF annual reviews from 2013. Currently no guidelines exist as to the frequency of CPET in CF patients.

Aims To determine whether CPET was a useful and feasible routine test in children with $\mathrm{CF}$ and to assess whether VO2 peak correlated with Forced Expiratory Volume in $1 \mathrm{~s}$ (FEV1), disease severity, gender, genotype or previous intravenous antibiotics.

Method A pilot observational study was performed. Body mass index (BMI) was used as a marker of disease severity. Genotype was divided into 3 groups; DF508 homozygous, heterozygous and all other genotypes. Data was retrieved from case notes and our CF database.

Results 38 patients (17 male, 21 female) underwent CPET. Age range 7-14 (mean 9.8) years. 36/38 had technically satisfactory CPET. Mean VO2 peak was $107 \%$ predicted, standard deviation $18 \%$. Only $8 \%$ had an abnormal VO2 peak. Mean FEV1 was 91\% predicted, standard deviation $15 \%$. There was no relationship between VO2 peak and FEV1 [p = 0.297] or BMI [p = 0.382] (Pearson correlation). Additionally, no correlation was demonstrated between VO2 peak and genotype $[\mathrm{p}=0.236]$ (one-way ANOVA), gender $[\mathrm{p}=0.902]$ or patients who had received at least one course of intravenous antibiotics in the past year $[\mathrm{p}=0.253$ ] (two-sample T test).

Conclusions CPET is a feasible test with $95 \%$ of our patients achieving technically satisfactory assessments and reassuringly, VO2 peak was largely normal. We could not demonstrate a relationship with FEV1 or disease severity although our study is limited by the small sample size. It is recognised that the CF annual review is already a long day for patients and we plan to reserve CPET for those showing exercise intolerance rather than performing it annually.

\section{REFERENCES}

1 Rogers D, Prasad SA, Doull I. Exercise testing in children with cystic fibrosis. J Royal Society Med 2003;96:23-29

2 Nixon PA, Orenstein DM, Kelsey SF, et al. The prognostic value of exercise testing in patients with cystic fibrosis. N Engl J Med 1992;327:1785-88

3 American Thoracic Society/American College of Chest Physicians. ATS/ACCP statement on cardiopulmonauy exercise testing. Am J Respir Crit Care Med 2003; 167:211-77

\section{G378 DEMOGRAPHICS AND SELECTED CLINICAL FEATURES OF PAEDIATRIC HUMAN METAPNEUMOVIRUS INFECTION}

${ }^{1} Y$ Petrunin, ${ }^{2} \mathrm{~L}$ Jones, ${ }^{3} \mathrm{~K}$ Templeton, ${ }^{2} \mathrm{D}$ Urquhart. ${ }^{1}$ University of Edinburgh, Edinburgh, UK; ${ }^{2}$ Department of Paediatrics, Royal Hospital for Sick Children, Edinburgh, UK; ${ }^{3}$ Department of Virology, Royal Infirmary of Edinburgh, Edinburgh, UK

\subsection{6/archdischild-2015-308599.333}

Aims This study aimed to characterise seasonal variation, coinfection rates, susceptible groups (by gender, age and co-morbidities) and selected clinical features of childhood human metapneumovirus (HMPV) infection.

Methods The study was a retrospective analysis of 656 HMPVpositive respiratory samples collected from paediatric patients aged $0-15$ years processed from January 2010 to December 2013 using real time PCR assays. In addition, 200 HMPV-positive samples from January 2012 to January 2013 were cross-referenced with electronic discharge summaries and descriptive statistical analyses of selected clinical features were performed.

Results 377 of 656 (57\%) HMPV-positive samples were from male patients. HMPV was most frequently detected in children aged 6-9 months and the median age of patients studied was 15 months. Seasonal patterns of HMPV infection varied from year to year. The peak in HMPV-positive isolates occurred between February and May. 117 of 656 (17.5\%) samples were positive for other respiratory viruses in addition to HMPV. The most common co-infections were due to rhinovirus $(58 / 656,8.84 \%)$ and adenovirus $(36 / 656,5.49 \%)$.

$81(40 \%)$ of the 200 fully-characterised patients were hospitalised, 7 (3.5\%) of whom required intensive care (ICU) or high dependency (HDU) admission. Rashes were reported in 14 (7\%) and febrile seizures in $9(4.5 \%)$ of 200 patients. These clinical features were more common in children with co-infections $[6 / 40$ (15\%) and 4/40 (10\%) respectively].

Conclusions Male gender is suggested as a predisposing factor for HMPV infection, along with younger age. Seasonal variation of HMPV infection in Scottish children appears different to the reported winter peaks of other studies, with peak incidence occurring between February and May in our dataset. Co-infection is common, and most frequently associated with rhinovirus or adenovirus. Rashes and febrile seizures are relatively common in HMPV-positive patients, especially those co-infected with other respiratory pathogens. Human metapneumovirus is a significant cause of morbidity in children. Further, larger-scale epidemiological research appears warranted, along with work to develop new therapies aimed at targeting HMPV.

\section{G379 DESCRIPTIVE ANALYSIS OF ADHERENCE WITH NON- INVASIVE VENTILATION IN CHILDREN}

MJ Vincent, P Davies, N Gibson, A Morley. Paediatric Respiratory Medicine, Royal Hospital for Sick Children, Glasgow, UK

\subsection{6/archdischild-2015-308599.334}

Aims Home non-invasive ventilation (NIV) is an established treatment for children with sleep-disordered breathing. There is 